\title{
Amino-functionalized (meth)acryl polymers by use of a solvent-polarity sensitive protecting group (Br-t-BOC)
}

\author{
Helmut Ritter ${ }^{*}$, Monir Tabatabai and Markus Herrmann
}

\author{
Full Research Paper \\ Address: \\ Institute of Organic Chemistry and Macromolecular Chemistry, \\ Heinrich-Heine-University Duesseldorf, Universitaetsstraße 1, \\ D-40225 Duesseldorf, Germany \\ Email: \\ Helmut Ritter ${ }^{*}$ - h.ritter@uni-duesseldorf.de \\ * Corresponding author \\ Keywords: \\ amino group protection; bromo-tert-butyloxycarbonyl; deprotection; \\ free radical polymerization; (meth)acryl polymers; neighboring group \\ effects; solvent polarity
}

\author{
Beilstein J. Org. Chem. 2016, 12, 245-252. \\ doi:10.3762/bjoc. 12.26 \\ Received: 25 November 2015 \\ Accepted: 25 January 2016 \\ Published: 10 February 2016 \\ Associate Editor: P. J. Skabara \\ (C) 2016 Ritter et al; licensee Beilstein-Institut. \\ License and terms: see end of document.
}

\begin{abstract}
We describe the synthesis of bromo-tert-butyloxycarbonyl (Br-t-BOC)-amino-protected monomers 2-((1-bromo-2-methylpropan-2yl)oxycarbonylamino)ethyl (meth)acrylate 3a,b. For this purpose, 2-isocyanatoethyl (meth)acrylate 1a,b was reacted with 1-bromo2-methylpropan-2-ol (2a). The free radical polymerization of (Br-t-BOC)-aminoethyl (meth)acrylates 3a,b yielded poly((Br-tBOC)-aminoethyl (meth)acrylate) 6a,b bearing protected amino side groups. The subsequent solvolysis of the Br-t-BOC function led to the new polymers poly(2-aminoethyl (meth)acrylate) 8a,b with protonated free amino groups. The monomers and the resulting polymers were thoroughly characterized by ${ }^{1} \mathrm{H}$ NMR, IR, GPC and DSC methods. The kinetics of the deprotection step was followed by ${ }^{1} \mathrm{H}$ NMR spectroscopy. The solvent polarity and neighboring group effects on the kinetics of deprotection are discussed.
\end{abstract}

\section{Introduction}

Amino groups are important functionalities in polymer chemistry, e.g., for hardening various epoxy resins [1]. However, they easily react in an undesired side reaction with electronpoor double bonds of (meth)acrylates [2]. Therefore, for the synthesis of amino-containing (meth)acrylic monomers and polymers suitable amino-protecting groups are required. Classical protecting groups such as ammonium salts, F-MOC, Z- or $t$-BOC, respectively are readily available. Regarding to this aspect, we published some papers about polymer protecting groups about three decades ago [3-7]. However, they are limited in application by certain restrictions on the deprotection conditions. Therefore, there is a continued interest in developing new protecting groups which can be cleaved by different mechanisms. Keeping this in mind, the bromo-tert-butyloxycarbonyl (Br-t-BOC) group represents the first known solvent-polarity sensitive amino-protecting group. As shown in Figure 1, this group is stable in nonpolar solvents because of high activation energy and easily decomposes in a more polar environment because of reduced activation energy. This effect is a result of an increased polarity of the transition state in comparison to the 


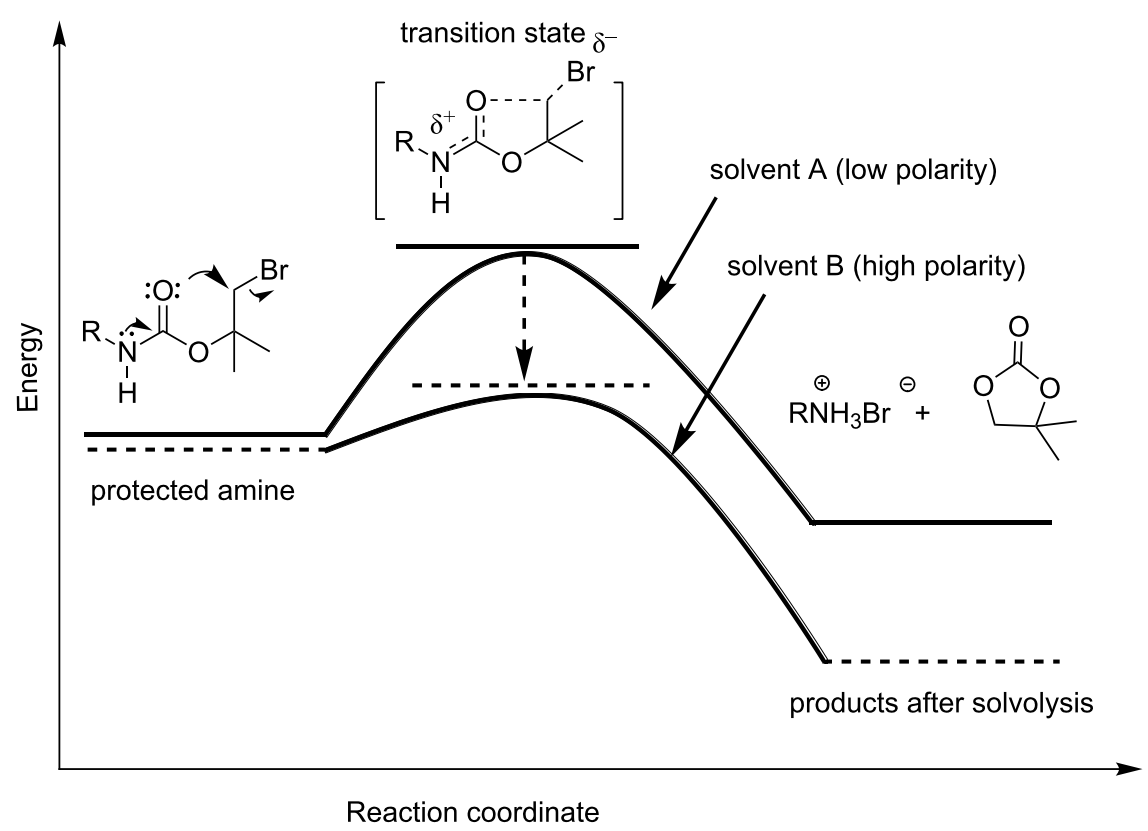

Figure 1: Schematic representation of a deprotection taking the relatively high polarity of the transition state and the solvent polarity into account: high activation energy in nonpolar solvents and low activation energy in polar solvents.

starting molecule (Figure 1). In contrast to the classical $t$-BOC protecting group, the $\mathrm{Br}-t$-BOC group can be easily removed without $\mathrm{pH}$ adjustments. Since the published papers from L. A. Carpino [8-10] and a first practical application in peptide synthesis [11] this protecting group was quasi forgotten.

In the present work we report new Br-t-Boc-protected (meth)acrylic monomers and their polymerization through free radical polymerization. The kinetics of $\mathrm{Br}-t$-BOC solvolysis of the monomers and polymers in a polar solvent are described.

\section{Results and Discussion}

The Br- $t$-Boc-protected monomers 2-((1-bromo-2-methylpropan-2-yl)oxycarbonylamino)ethyl acrylate (3a) and 2-((1bromo-2-methylpropan-2-yl)oxycarbonylamino)ethyl methacrylate (3b) were synthesized by the reaction of 1-bromo-2-methylpropan-2-ol (2a) with 2-isocyanatoethyl (meth)acrylate (1a,b) (Scheme 1). The success of the reaction can be easily shown by, e.g., the disappearance of the $\mathrm{N}=\mathrm{C}=\mathrm{O}$ peak of $\mathbf{1 a}$ at $2260 \mathrm{~cm}^{-1}$ and the appearance of an $\mathrm{N}-\mathrm{H}$ peak at $3350 \mathrm{~cm}^{-1}$ in the IR spectra. Pure samples of $\mathbf{3 a}, \mathbf{b}$ could be isolated by column chro-<smiles>[R]C(=C)C(=O)OCCN=C=O</smiles>

1a,b<smiles>CC(C)(O)CBr</smiles>

2a<smiles>[R]C(=C)C(=O)OCCNC(=O)OC(C)(C)CBr</smiles>

3a,b

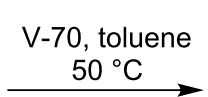<smiles>[R]C(CC(C)(C)C)(C(=O)OCCNC(=O)OC(C)(C)CBr)C(C)(C)CBr</smiles> 
matography and the ${ }^{1} \mathrm{H}$ NMR spectra of $\mathbf{3 a}$ and $\mathbf{3} \mathbf{b}$ are shown in Figure 2 and Figure 3.

After dissolving monomers $\mathbf{3 a}$ and $\mathbf{3 b}$ in a polar solvent, they show the above mentioned self-cleaving process yielding the corresponding amine hydrobromide $(\mathbf{4 a}, \mathbf{b})$ and the cyclic carbonate 4,4-dimethyl-1,3-dioxolan-2-one (5). As explained above, the protecting group undergoes a polar solvent driven intramolecular nucleophilic displacement (Figure 1). In contrast, in nonpolar solvents such as benzene, toluene, chloro-

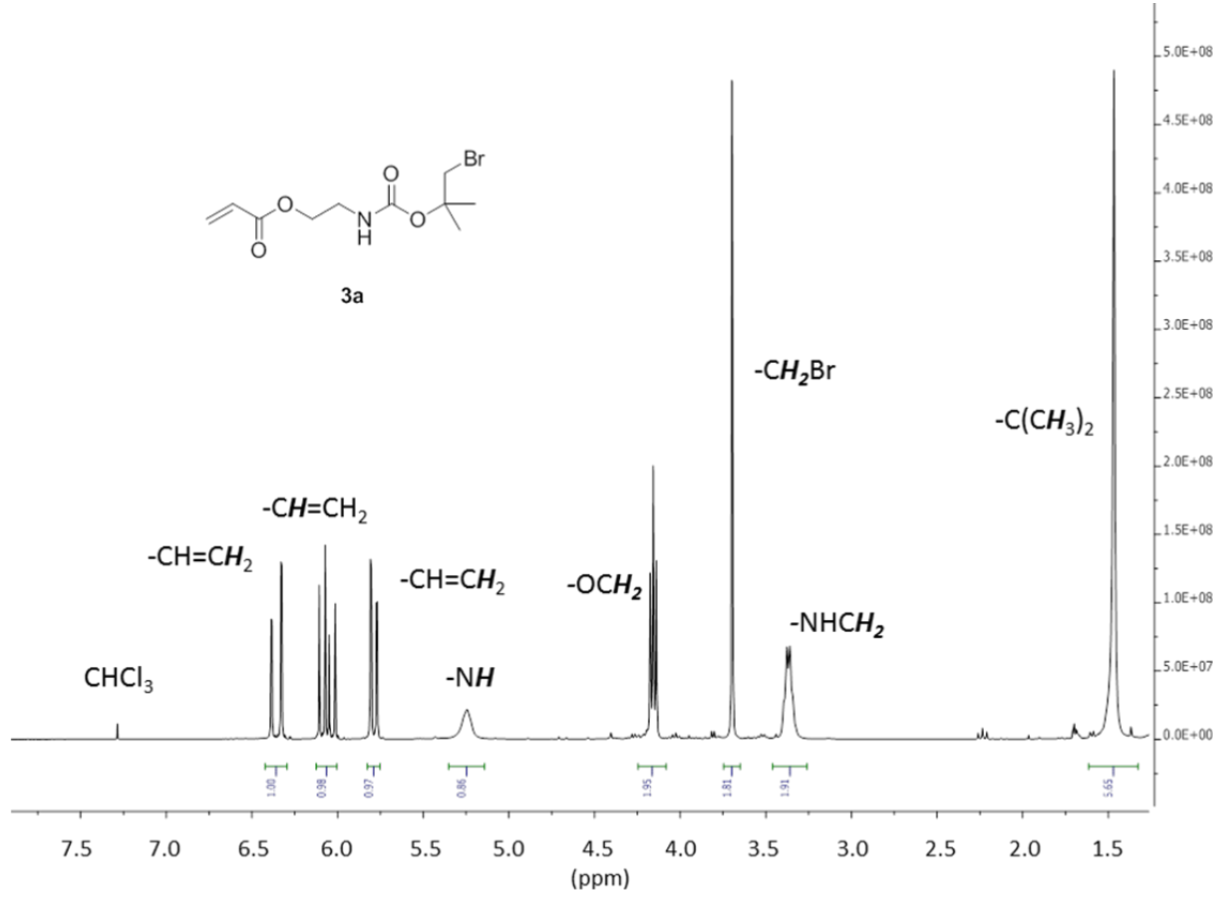

Figure 2: $300 \mathrm{MHz}{ }^{1} \mathrm{H}$ NMR spectrum of $3 \mathbf{a}$ in $\mathrm{CDCl}_{3}$.

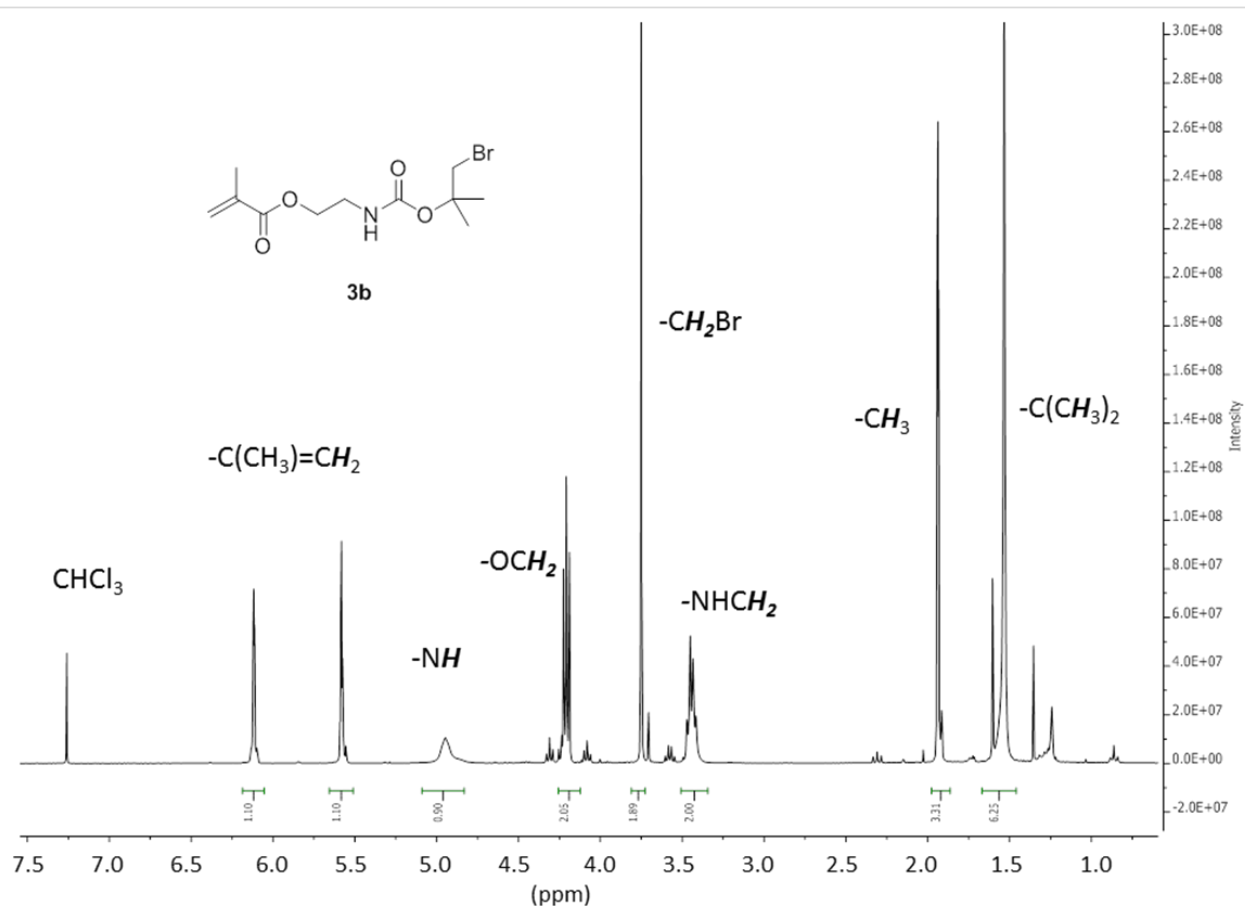

Figure 3: $300 \mathrm{MHz}{ }^{1} \mathrm{H}$ NMR spectrum of $\mathbf{3 b}$ in $\mathrm{CDCl}_{3}$. 
form $\left(\mathrm{CHCl}_{3}\right)$ or dichloromethane $\left(\mathrm{CH}_{2} \mathrm{Cl}_{2}\right) \mathbf{3 a}, \mathbf{b}$ are very stable, whereas in polar protic solvents such as ethanol (EtOH) or methanol $(\mathrm{MeOH})$ the cleaving takes place very fast (Scheme 2). This deprotecting process can be directly followed using ${ }^{1} \mathrm{H}$ NMR spectroscopy.

Carpino assumed that, changing one or both of the methyl groups to bulkier substituents would significantly increase the cleaving process [10]. Thus, we attempted to replace one methyl group with a phenyl substituent simply by the reaction of 1a with 1-bromo-2-phenylpropan-2-ol (2b). Although spectroscopic examinations gave evidence for the successful formation of the expected carbamate $\mathbf{3 c}$, only the corresponding amine hydrobromide $4 \mathbf{a}$ was isolated as main product. This means that the amino-protecting group is extremely sensitive to decomposition. A third type of monomer was synthesized by reaction of 1a with 1-bromo-2-methylbut-3-en-2-ol (2c) yielding 2-((1-bromo-2-methylbut-3-en-2-yl)oxycarbonylamino)ethyl acrylate (3d, Scheme 3). Although monomer 3d is stable in nonpolar solvents, the cleaving process could not be followed by ${ }^{1} \mathrm{H}$ NMR spectroscopy because of rapid decomposition in a polar solvent.

Next, classical free radical polymerizations of $\mathbf{3 a}, \mathbf{b}$ were carried out in toluene as solvent at $50{ }^{\circ} \mathrm{C}$ using 2,2'-azobis(4-methoxy2,4-dimethylvaleronitrile) ( $\mathrm{V}-70)$ as initiator yielding homopolymers $\mathbf{6 a}, \mathbf{b}$. This initiator is known to have a low decomposition temperature of $30^{\circ} \mathrm{C}$ in toluene. Additionally, copolymers of $\mathbf{3 a}$ and $\mathbf{3 b}$, respectively with $N, N$-dimethylacrylamide (7) were synthesized yielding $\mathbf{7 a}, \mathbf{b}$. It was assumed that, since the cleaving process is coupled with cyclization to create the cyclic carbonate $\mathbf{5}$, the homopolymer could be self-deactivated due to competing hydrogen-bond interactions of the urethane carbonyl group with neighboring groups. Since the comonomer should act as diluting agent a better access of the carbonyl group to the bromo-substituted carbon atom to fulfill the nucleophilic intramolecular displacement could be expected (Scheme 4).

The kinetic studies of 3a, 6a and 7a are shown in Figure 4. Additionally, Figure 5 shows the ${ }^{1} \mathrm{H}$ NMR spectra of homopolymer $6 \mathbf{a}$ and deprotected polymer $\mathbf{8 a}$.

The calculated $t_{0.5}$ and $t_{0.3}$ values of deprotection for $\mathbf{3 a}, \mathbf{b}, \mathbf{6 a}, \mathbf{b}$ and $7 \mathbf{a}, \mathbf{b}$ are summarized in Table 1.

\begin{tabular}{|c|c|c|}
\hline Compound & $t_{0.3}[\mathrm{~min}]$ & $t_{0.5}[\mathrm{~min}]$ \\
\hline $3 a$ & 84 & 180 \\
\hline $3 b$ & 102 & 180 \\
\hline $6 a$ & 162 & 276 \\
\hline $6 b$ & 159 & 264 \\
\hline $7 a$ & 114 & 192 \\
\hline $7 b$ & 120 & 204 \\
\hline
\end{tabular}

As assumed above the molecular dispersed monomer shows the highest reactivity followed by the copolymer. The solvolysis of the protecting group in the homopolymer is relatively slow

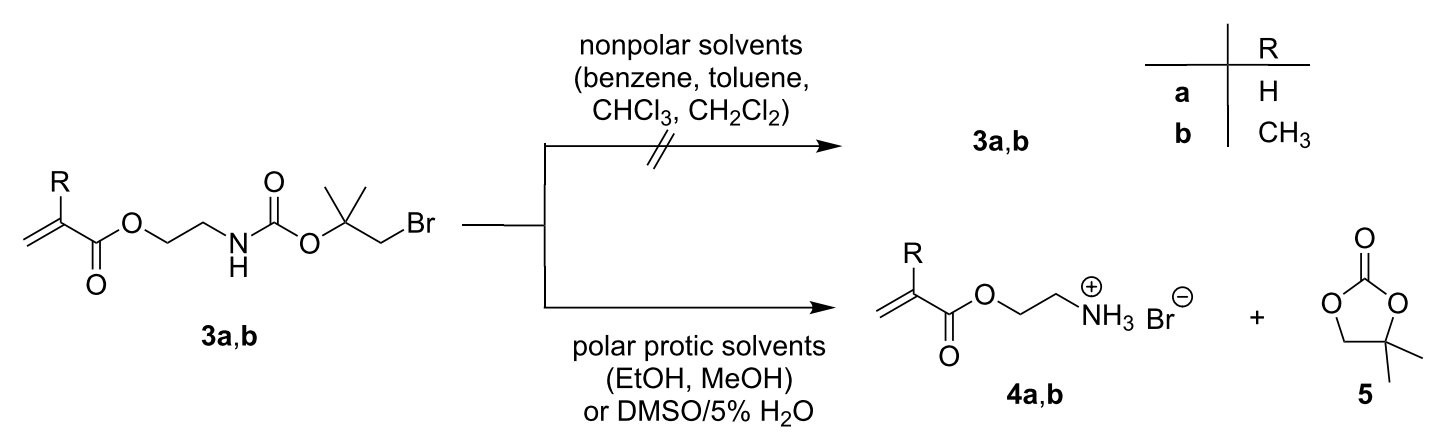

Scheme 2: Stability of $\mathbf{3}$ in different solvents.<smiles>[R]C(C)(CBr)OC(=O)NCCOC(=O)C=CCC</smiles>

\begin{tabular}{l|l} 
& \multicolumn{1}{|c}{$R$} \\
\hline 2b, 3c & $\mathrm{C}_{6} \mathrm{H}_{5}$ \\
2c, 3d & $\mathrm{CH}=\mathrm{CH}_{2}$
\end{tabular}


a)<smiles></smiles>

b)<smiles>[R]C(C)(CC)C(=O)OCCNC(=O)OC(C)(C)CBr</smiles>

$7 a, 7 b$

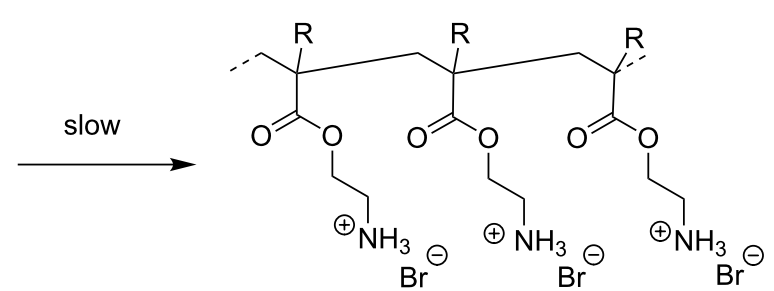

$8 a, 8 b$

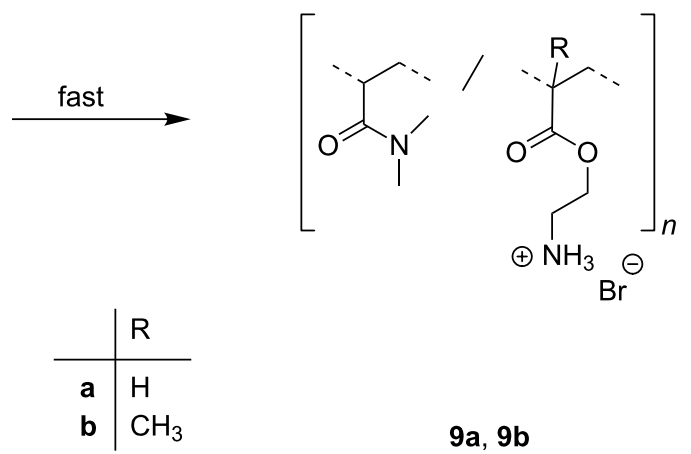

Scheme 4: a) Self deactivation of homopolymer $\mathbf{6 a}, \mathbf{b}$ due to competing hydrogen interactions in comparison to b) copolymer $7 \mathbf{a}, \mathbf{b}$.

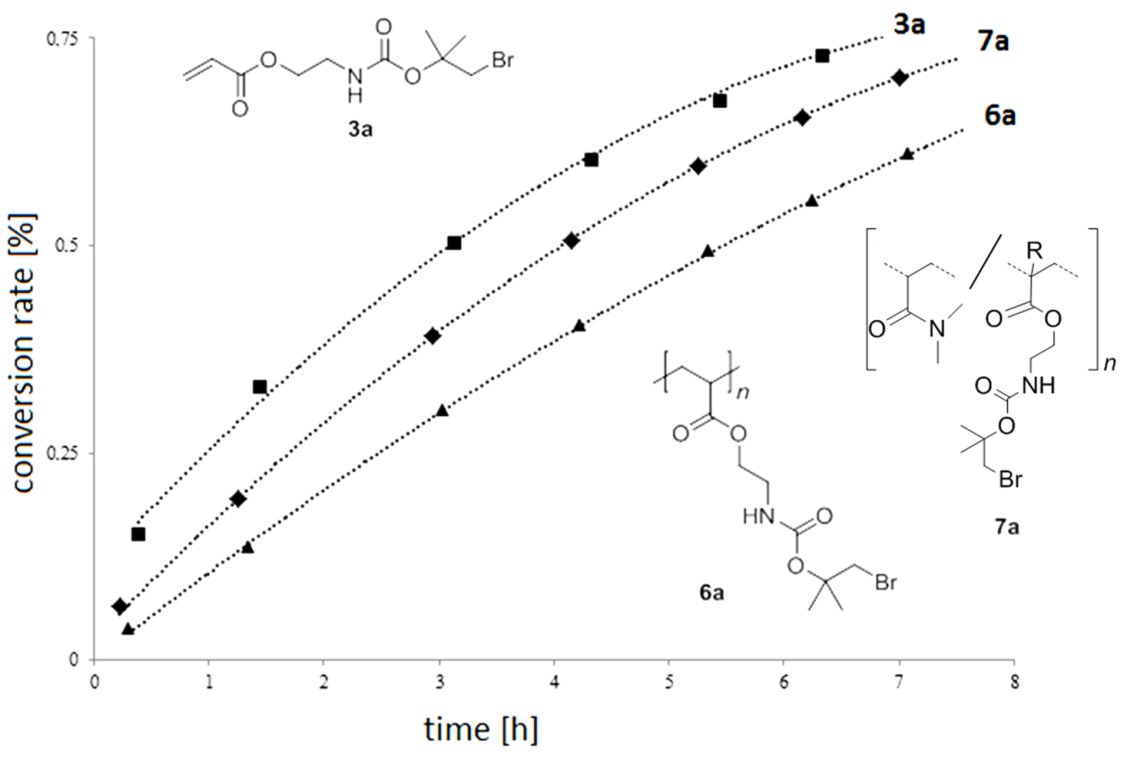

Figure 4: Kinetic studies of the deprotection of $3 \mathbf{a}, \mathbf{6} \mathbf{a}$ and $7 \mathbf{a}$.

because of neighboring group formed retarding hydrogen bonds (Scheme 4).

\section{Conclusion}

It can be concluded from the above described results that the bromo-tert-butyloxycarbonyl group has a certain potential for the preparation of amino group containing functional (meth)acryl polymers. Since the deprotection takes place under mild conditions in polar solvents, some sensitive components, e.g., drugs, proteins, DNA may be present without being affected.

\section{Experimental}

\section{Methods}

${ }^{1} \mathrm{H}$ NMR measurements were performed using a Bruker Avance 300 operating at $300 \mathrm{MHz}$ at room temperature. Fourier trans- 

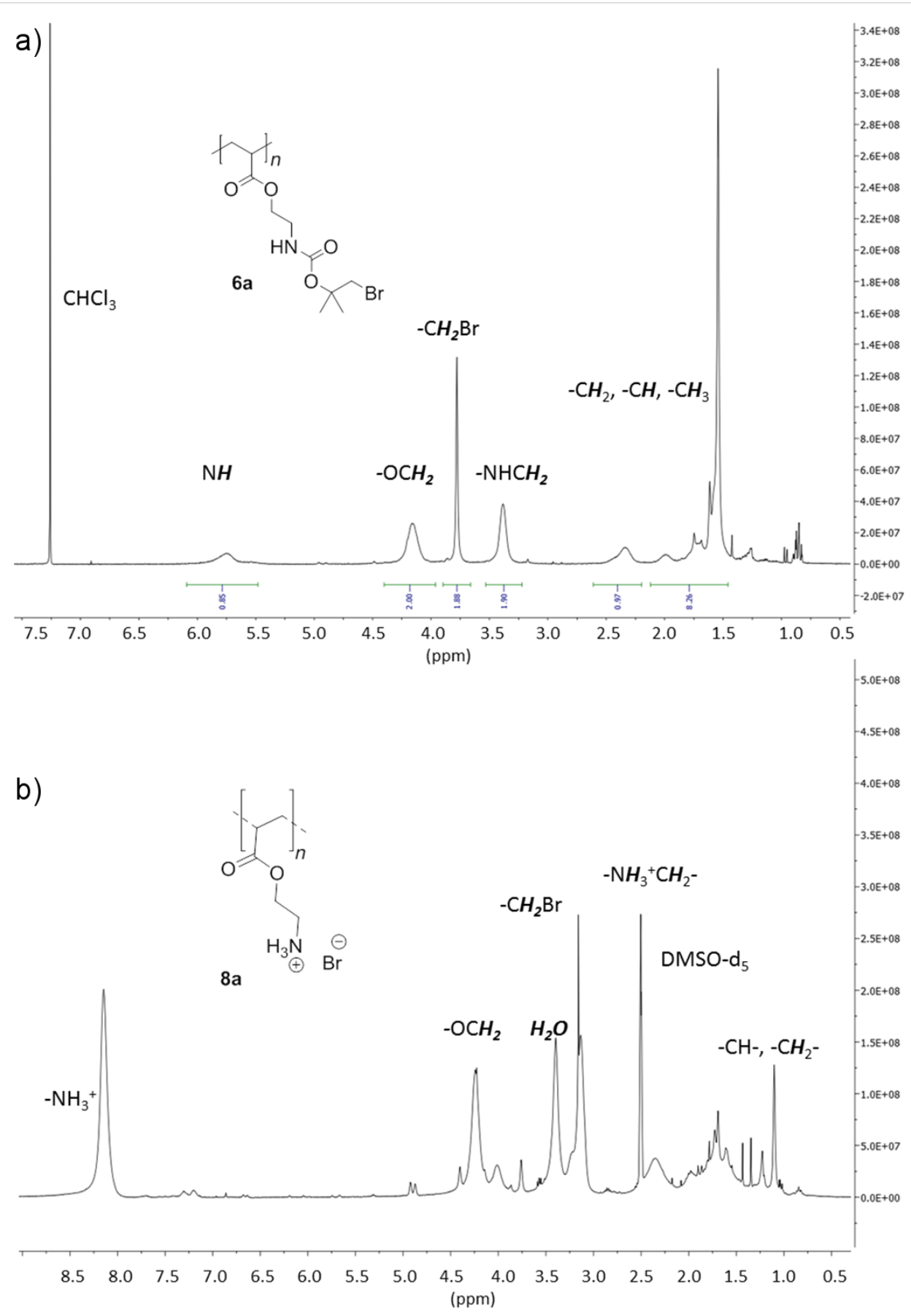

Figure 5: $300 \mathrm{MHz}{ }^{1} \mathrm{H}$ NMR spectra of a) $\mathbf{6 a}$ in $\mathrm{CDCl}_{3}$ and b) $8 \mathbf{a}$ in DMSO- $d_{6}$.

formation infrared spectroscopy (FTIR) was performed on a Nicolet 6700 FTIR spectrometer equipped with a diamond single bounce ATR accessory. The measurements were performed in the range of $400-3000 \mathrm{~cm}^{-1}$ at room temperature. Differential scanning calorimetry (DSC) was performed using a Mettler Toledo DSC 822 instrument equipped with a sample robot TSO801RO. The Apparatus was controlled over a temperature range between $-50{ }^{\circ} \mathrm{C}$ and $350{ }^{\circ} \mathrm{C}$ at a heating rate of $15^{\circ} \mathrm{C} \min ^{-1}$. The $T_{\mathrm{g}}$ values are reported as the average of five measurements using the midpoint method. The THF-GPC System compromises a Scharmbeck SFD degasser (Gastor BG12), a FLOW pump (Intelligent PUMP AL-12) and a Scharmbeck SFD (Model S5200) sampler. A Waters 486 Turnable Absorbance Detector and a Scharmbeck SFD RI 2000 detector were used for detection. A set of columns packed with porous styrene-divinylbenzene-copolymer beads was used for 
separation of the analytes (MZ Analysentechnik GmbH, $1 \times$ guard column $100 \AA, 3 \times$ columns with $10,000,1,000$ und $100 \AA$ ). The system was calibrated with polystyrene standards with a molecular range from $575 \mathrm{~g} / \mathrm{mol}$ to $3,114,000 \mathrm{~g} / \mathrm{mol}$.

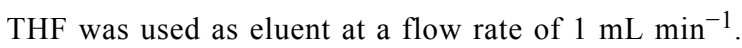

\section{Materials}

Commercial reagents and solvents were purchased from SigmaAldrich, Merck and Fluka. If not stated otherwise all chemicals were of analytical grade and were used as received without any further purification. 2-Isocyanatoethyl acrylate and (meth)acrylate were purchased from Shōwa Denkō K.K.

Chloroform- $d$ (99.8 atom \% D) was obtained from Deutero GmbH (Germany). All solvents were dried by standard methods. Column chromatography was performed using Acros Organics silica gel 60 (230-400 mesh) and thin layer chromatography (TLC) of the products using Merck silica 60 F254 plates.

\section{1-Bromo-2-methylpropan-2-ol (2a)}

In a $500 \mathrm{~mL}$ three-necked flask with a reflux condenser tert-butanol (100 g, $1.35 \mathrm{~mol}$ ) was heated under reflux. A second $500 \mathrm{~mL}$ single-necked flask was equipped with $\mathrm{N}$-bromosuccinimide (NBS, $50 \mathrm{~g} 0.28 \mathrm{~mol}$ ) in a mixture of $\mathrm{THF} / \mathrm{H}_{2} \mathrm{O} 1: 2$. To the boiling tert-butanol 5 portions of $5 \mathrm{~mL}$ each concentrated sulfuric acid were added dropwise every $15 \mathrm{~min}$. The forming isobutene was bubbled into the NBS solution under vigorous stirring. After $45 \mathrm{~min}$, all of the NBS disappeared and stirring was continued for an additional hour. THF was evaporated and the remaining aqueous mixture was extracted several times with diethyl ether. The extract was dried over magnesium sulfate and the solution was evaporated. The remaining oil was distilled to give 2a as a pure colorless liquid: yield $22 \mathrm{~g}(51 \%)$; bp $55^{\circ} \mathrm{C}$ at 25 mbar; ${ }^{1} \mathrm{H}$ NMR $\left(300 \mathrm{MHz}, \mathrm{CDCl}_{3}\right) \delta[\mathrm{ppm}] 3.39(\mathrm{~s}, 2 \mathrm{H}$, - $\mathrm{CH}_{2}$ - $\mathrm{Br}$ ); 2.43 (s, $\left.1 \mathrm{H},-\mathrm{OH}\right) ; 1.30$ (s, 6H, - $\mathrm{CH}_{3}$ ); IR (diamond) $\tilde{v}\left[\mathrm{~cm}^{-1}\right]: 3380\left(s, v_{\mathrm{OH}}\right), 2976\left(m, v_{\mathrm{C}-\mathrm{H}}\right), 2931\left(m, v_{\mathrm{C}-\mathrm{H}}\right), 2872$ $\left(m, v_{\mathrm{C}-\mathrm{H}}\right), 1465\left(m, v_{-\mathrm{CH} 2-)}, 1379\left(s, v_{\mathrm{OH}}\right)\right.$.

\section{1-Bromo-2-phenylpropan-2-ol (2b)}

Into a stirring solution of $\mathrm{N}$-bromosuccinimide (NBS, $32 \mathrm{~g}$, $0.18 \mathrm{~mol}$ ) in $1 / 3$ THF and $2 / 3 \mathrm{H}_{2} \mathrm{O}, \alpha$-methylstyrene $(10 \mathrm{~mL}$, $0.15 \mathrm{~mol}$ ) were added. After $2 \mathrm{~h}$ of vigorous stirring, THF was evaporated and the aqueous solution was extracted several times with diethyl ether. The extract was dried over magnesium sulfate and the solution was evaporated. The crude product was purified by column chromatography using $n$-hexane/ethyl acetate 1:1 to give the desired colorless liquid $\mathbf{2 b}$ : yield $21.35 \mathrm{~g}$ (66\%). ${ }^{1} \mathrm{H}$ NMR $\left(300 \mathrm{MHz}, \mathrm{CDCl}_{3}\right) \delta[\mathrm{ppm}] 7.57-7.54(\mathrm{~m}, 2 \mathrm{H}$, $\operatorname{Ar} H), 7.38-7.32(\mathrm{~m}, 2 \mathrm{H}, \operatorname{Ar} H), 7.27-7.22(\mathrm{~m}, 1 \mathrm{H}, \operatorname{Ar} H), 5.40$ $(\mathrm{s}, 1 \mathrm{H},-\mathrm{OH}), 3.78\left(\mathrm{~s}, 2 \mathrm{H},-\mathrm{CH}_{2}-\mathrm{Br}\right), 1.66\left(\mathrm{~s}, 3 \mathrm{H},-\mathrm{CH}_{3}\right)$; IR (diamond) $\tilde{v}\left[\mathrm{~cm}^{-1}\right]: 3442\left(\mathrm{~s}, v_{\mathrm{OH}}\right), 3027\left(\mathrm{~m}, v_{\mathrm{C}-\mathrm{H}}\right), 2978(\mathrm{~m}$, $\left.v_{\mathrm{C}-\mathrm{H}}\right), 1602\left(w, v_{\mathrm{C}=\mathrm{C}}\right), 1493\left(w, v_{\mathrm{C}=\mathrm{C}}\right), 1446\left(m, v_{-\mathrm{CH} 2-}\right), 1374$ $\left(s, v_{\mathrm{OH}}\right.$ tert-alcohol $)$.

\section{1-Bromo-2-methylbut-3-en-2-ol (2c)}

Into a stirring solution of $\mathrm{N}$-bromosuccinimide (NBS, $20 \mathrm{~g}$, $0.12 \mathrm{~mol}$ ) in $1 / 3$ THF and $2 / 3 \mathrm{H}_{2} \mathrm{O}$, isoprene was added in 3 small portions of $5 \mathrm{~mL}(0.05 \mathrm{~mol})$ at $0{ }^{\circ} \mathrm{C}$. Stirring was continued for an additional hour. THF was evaporated and the remaining aqueous mixture was extracted several times with diethyl ether. The extract was dried over magnesium sulfate and the solution was evaporated. The remaining oil was distilled to give 2c as a pure colorless liquid: yield $7.41 \mathrm{~g}$ (37.5\%); bp $62{ }^{\circ} \mathrm{C}$ at 20 mbar. ${ }^{1} \mathrm{H}$ NMR $\left(300 \mathrm{MHz}, \mathrm{CDCl}_{3}\right) \delta[\mathrm{ppm}] 5.88$ $(\mathrm{dd}, 1 \mathrm{H},-\mathrm{CH}=\mathrm{CH}), 5.34-5.16\left(\mathrm{~m}, 2 \mathrm{H},-\mathrm{CH}=\mathrm{CH}_{2}\right), 3.44(\mathrm{~s}$, $\left.2 \mathrm{H},-\mathrm{C} H_{2}-\mathrm{Br}\right), 2.32(\mathrm{~s}, 1 \mathrm{H},-\mathrm{OH}), 1.40\left(\mathrm{~s}, 3 \mathrm{H},-\mathrm{CH}_{3}\right)$; IR (diamond) $\tilde{v}\left[\mathrm{~cm}^{-1}\right]: 3412\left(\mathrm{~s}, v_{\mathrm{OH}}\right), 2980\left(\mathrm{~m}, v_{\mathrm{C}-\mathrm{H}}\right), 2928(\mathrm{~m}$, $\left.v_{\mathrm{C}-\mathrm{H}}\right), 1644\left(m, v_{\mathrm{C}=\mathrm{C}}\right), 1453\left(m, v_{-\mathrm{CH} 2-}\right), 1371\left(s, v_{\mathrm{OH}}\right)$.

\section{General information for the preparation of the monomers}

All syntheses were carried out under an argon atmosphere at room temperature using dibutyltin dilaurate (DBTL) as catalyst.

\section{2-((1-Bromo-2-methylpropan-2-yl)oxy- carbonylamino)ethyl acrylate (3a)}

To a stirring solution of 2-isocyanatoethyl acrylate (1a, $8 \mathrm{~g}$, $0.056 \mathrm{~mol}$ ) and $2 \mathrm{~mol} \% \mathrm{DBTL}$ in $25 \mathrm{~mL}$ toluene, 1-bromo-2methylpropan-2-ol (2a, 7.7 g, 0.05 mol) was added. After $24 \mathrm{~h}$ the solvent was evaporated and the crude product was purified by column chromatography using $n$-hexane/ethyl acetate $1: 1$ to give the pure product 3a: yield $5.39 \mathrm{~g}(32 \%)$. ${ }^{1} \mathrm{H}$ NMR $\left(300 \mathrm{MHz}, \mathrm{CDCl}_{3}\right) \delta[\mathrm{ppm}] 6.32\left(\mathrm{dd},{ }^{3} J=17.3 \mathrm{~Hz},{ }^{2} J=1.5 \mathrm{~Hz}\right.$, $1 \mathrm{H},-\mathrm{CH}=\mathrm{CHH}), 6.04\left(\mathrm{dd},{ }^{3} J=17.3 \mathrm{~Hz},{ }^{3} J=10.4 \mathrm{~Hz}, 1 \mathrm{H}\right.$, $\left.-\mathrm{CH}=\mathrm{CH}_{2}\right), 5.76\left(\mathrm{dd},{ }^{3} \mathrm{~J}=10.4 \mathrm{~Hz},{ }^{2} \mathrm{~J}=1.5 \mathrm{~Hz}, 1 \mathrm{H}\right.$, $-\mathrm{CH}=\mathrm{CHH}), 5.22(\mathrm{~s}, 1 \mathrm{H},-\mathrm{NH}-), 4.13\left(\mathrm{t},{ }^{3} J=5.4 \mathrm{~Hz}, 2 \mathrm{H}\right.$, $-\mathrm{OCH}_{2}$ ), 3.67 (s, 2H, - $\left.\mathrm{CH}_{2}-\mathrm{Br}\right), 3.34$ (q, ${ }^{3} J=5.6 \mathrm{~Hz}, 2 \mathrm{H},-\mathrm{NH}-$ $\left.\mathrm{CH}_{2}\right), 1.44\left(\mathrm{~s}, 6 \mathrm{H},-\mathrm{CH}_{3}\right)$; IR (diamond) $\tilde{v}\left[\mathrm{~cm}^{-1}\right]: 3358(\mathrm{~m}$, $\left.v_{\mathrm{N}-\mathrm{H}}\right), 2982\left(m, v_{\mathrm{C}-\mathrm{H}}\right), 2936\left(m, v_{\mathrm{C}-\mathrm{H}}\right), 1708\left(w, v_{\mathrm{C}=\mathrm{O}}\right), 1635(w$, $\left.v_{\mathrm{C}=\mathrm{C}}\right), 1618\left(w, v_{\mathrm{C}=\mathrm{Ct}}\right), 1516\left(s, v_{\mathrm{N}-\mathrm{H}}\right), 983\left(s, v_{\mathrm{CH}=\mathrm{CH} 2}\right), 809$ $\left(s, v_{\mathrm{CH}=\mathrm{CH} 2}\right)$.

\section{2-((1-Bromo-2-methylpropan-2-yl)oxy- carbonylamino)ethyl methacrylate (3b)}

To a stirring solution of 2-isocyanatoethyl methacrylate (1), $6 \mathrm{~g}, 0.039 \mathrm{~mol}$ ) and $2 \mathrm{~mol} \%$ DBTL in $25 \mathrm{~mL}$ toluene, 1-bromo2-methylpropan-2-ol (2a, $5.8 \mathrm{~g}, 0.038 \mathrm{~mol})$ was added. After $24 \mathrm{~h}$ the solvent was evaporated and the crude product was purified by column chromatography using $n$-hexane/ethyl acetate 1:1 to give the pure product $3 \mathbf{b}$ : yield $5.3 \mathrm{~g}(43 \%)$. ${ }^{1} \mathrm{H}$ NMR $\left(300 \mathrm{MHz}, \mathrm{CDCl}_{3}\right) \delta[\mathrm{ppm}] 6.12-6.11\left(\mathrm{~m}, 1 \mathrm{H},-\mathrm{C}\left(\mathrm{CH}_{3}\right)-\mathrm{CH}\right)$, 
5.59-5.57 (m, 1H, $\left.-\mathrm{C}\left(\mathrm{CH}_{3}\right)=\mathrm{CH} H\right), 4.95(\mathrm{~s}, 1 \mathrm{H},-\mathrm{NH}-), 4.21(\mathrm{t}$, $\left.{ }^{3} J=5.4 \mathrm{~Hz}, 2 \mathrm{H},-\mathrm{OCH}_{2}\right), 3.75\left(\mathrm{~s}, 2 \mathrm{H},-\mathrm{CH}_{2}-\mathrm{Br}\right), 3.44(\mathrm{~m}, 2 \mathrm{H}$, $\left.-\mathrm{NH}-\mathrm{CH}_{2}-\right), 1.93\left(\mathrm{~s}, 3 \mathrm{H},-\mathrm{CH}_{3}\right), 1.53\left(\mathrm{~s}, 6 \mathrm{H},-\mathrm{CH}_{3}\right)$; IR (diamond) $\tilde{v}\left[\mathrm{~cm}^{-1}\right]: 3365\left(\mathrm{~m}, v_{\mathrm{N}-\mathrm{H}}\right), 2978\left(\mathrm{~m}, v_{\mathrm{C}-\mathrm{H}}\right), 2929(\mathrm{~m}$, $\left.v_{\mathrm{C}-\mathrm{H}}\right), 1708\left(w, v_{\mathrm{C}=\mathrm{O}}\right), 1637\left(w, v_{\mathrm{C}=\mathrm{C}}\right), 1520\left(s, v_{\mathrm{N}-\mathrm{H}}\right), 943(s$, $\left.v_{\mathrm{CH}=\mathrm{CH} 2}\right), 814\left(s, v_{\mathrm{CH}=\mathrm{CH} 2}\right)$.

\section{2-((1-Bromo-2-methylbut-3-en-2-yl)oxy- carbonylamino)ethyl acrylate (3d)}

To a stirring solution of 2-isocyanatoethyl acrylate (1a, $4.27 \mathrm{~g}$, $0.03 \mathrm{~mol}$ ) and $2 \mathrm{~mol} \%$ DBTL in $25 \mathrm{~mL}$ toluene, 1-bromo-2methylbut-3-en-2-ol (2c, 5 g, $0.03 \mathrm{~mol})$ was added. After $24 \mathrm{~h}$ the solvent was evaporated and the crude product was purified by column chromatography using $n$-hexane/ethyl acetate $1: 1$ to give the pure product 3d: yield $1.7 \mathrm{~g}(17 \%) .{ }^{1} \mathrm{H}$ NMR $\left(300 \mathrm{MHz}, \mathrm{CDCl}_{3}\right) \delta[\mathrm{ppm}] 6.43\left(\mathrm{dd},{ }^{3} J=17,3 \mathrm{~Hz},{ }^{2} J=1.5 \mathrm{~Hz}\right.$, $1 \mathrm{H},-\mathrm{CH}-\mathrm{CHH}), 6.12\left(\mathrm{dd},{ }^{3} J=17.3 \mathrm{~Hz},{ }^{2} J=10.4 \mathrm{~Hz}, 2 \mathrm{H},-\mathrm{CH}\right.$ $\left.\mathrm{CH}_{2}\right), 5.86\left(\mathrm{dd},{ }^{3} J=10.4 \mathrm{~Hz},{ }^{2} J=1.5 \mathrm{~Hz}, 1 \mathrm{H},-\mathrm{CH}-\mathrm{CHH}\right)$, 5.81-5.74 (m, 1H, $\left.-\mathrm{CH}=\mathrm{CH}_{2}\right), 5.06(\mathrm{~s}, 1 \mathrm{H},-\mathrm{NH}-), 4.58-4.46$ $\left(\mathrm{m}, 2 \mathrm{H},-\mathrm{CH}=\mathrm{CH}_{2}\right), 4.24\left(\mathrm{t}, 2 \mathrm{H},-\mathrm{OCH}_{2}\right), 4.01-3.98(\mathrm{~m}, 2 \mathrm{H}$, $\left.-\mathrm{CH}_{2}-\mathrm{Br}\right), 3.50$ (q, 2H, -NH-CH $\left.\mathrm{C}_{2}\right), 1.73$ (s, $\left.3 \mathrm{H},-\mathrm{CH}_{3}\right)$; IR (diamond) $\tilde{v}\left[\mathrm{~cm}^{-1}\right]: 3348\left(\mathrm{~m}, v_{\mathrm{N}-\mathrm{H}}\right), 2951\left(\mathrm{~m}, v_{\mathrm{C}-\mathrm{H}}\right), 2869(\mathrm{~m}$, $\left.v_{\mathrm{C}-\mathrm{H}}\right), 1705\left(w, v_{\mathrm{C}=\mathrm{O}}\right), 1636\left(w, v_{\mathrm{C}=\mathrm{C}}\right), 1615\left(w, v_{\mathrm{C}=\mathrm{C}}\right), 1528(s$, $\left.v_{\mathrm{N}-\mathrm{H}}\right), 983\left(s, v_{\mathrm{CH}=\mathrm{CH} 2}\right), 808\left(s, v_{\mathrm{CH}=\mathrm{CH} 2}\right)$.

\section{General information for the preparation of the polymers and copolymers}

All polymerizations were carried out in toluene ( $75 \mathrm{wt} \%)$ at $50{ }^{\circ} \mathrm{C}$ using 5 mol \% 2,2'-azobis(4-methoxy-2,4-dimethylvaleronitrile) (V-70) as initiator. After $24 \mathrm{~h}$ the polymerizations were stopped and the solutions were precipitated by pouring into $n$-hexane. The obtained polymers were filtered off and dried under vacuum.

\section{Poly-(2-((1-bromo-2-methylpropan-2-yl)oxy- carbonylamino)ethyl acrylate) (6a)}

To a mixture of $\mathbf{3 a}(0.35 \mathrm{~g}, 1.19 \mathrm{mmol})$ in $0.7 \mathrm{~g}$ toluene, $\mathrm{V}-70$ ( $5 \mathrm{~mol} \%, 18.35 \mathrm{mg}$ ) dissolved in $0.35 \mathrm{~g}$ toluene was added. Polymer 6a was obtained as a colorless solid: yield $0.28 \mathrm{~g}$ (80\%). ${ }^{1} \mathrm{H}$ NMR $\left(300 \mathrm{MHz}, \mathrm{CDCl}_{3}\right) \delta[\mathrm{ppm}] 6.04-5.45(\mathrm{~m}$, $1 \mathrm{H}), 4.33-3.97(\mathrm{~m}, 2 \mathrm{H}), 3.90-3.60(\mathrm{~m}, 2 \mathrm{H}), 3.55-3.24(\mathrm{~m}, 2 \mathrm{H})$, 2.53-2.12 (m, 1H), 2.09-1.66 (m, 2H), 1.61-1.54 (m, 6H); IR (diamond) $\tilde{v}\left[\mathrm{~cm}^{-1}\right]: 3359\left(\mathrm{~m}, v_{\mathrm{N}-\mathrm{H}}\right), 2955\left(\mathrm{~m}, \mathrm{v}_{\mathrm{C}-\mathrm{H}}\right), 2930(\mathrm{~m}$, $\left.v_{\mathrm{C}-\mathrm{H}}\right), 1698\left(w, v_{\mathrm{C}=\mathrm{O}}\right), 1516\left(s, v_{\mathrm{N}-\mathrm{H}}\right)$; DSC: $T_{\mathrm{g}}=93.1^{\circ} \mathrm{C}$; GPC (THF): $\overline{M_{\mathrm{w}}}=9300 \mathrm{~g} / \mathrm{mol}, \overline{M_{\mathrm{n}}}=4400 \mathrm{~g} / \mathrm{mol}, \mathrm{D}=2.11$.

\section{Poly-(2-((1-bromo-2-methylpropan-2-yl)oxy- carbonylamino)ethyl methacrylate) (6b)}

To a mixture of $\mathbf{3 b}(0.38 \mathrm{~g}, 1.23 \mathrm{mmol})$ in $0.76 \mathrm{~g}$ toluene, $\mathrm{V}-70$ $(5 \mathrm{~mol} \%, 19.02 \mathrm{mg}$ ) dissolved in $0.38 \mathrm{~g}$ toluene was added. Polymer $6 \mathbf{b}$ was obtained as a colorless solid: yield $0.34 \mathrm{~g}$
(89\%). ${ }^{1} \mathrm{H}$ NMR (300 MHz, $\left.\mathrm{CDCl}_{3}, \mathrm{rt}\right) \delta[\mathrm{ppm}] 6.12-5.27(\mathrm{~m}$, $1 \mathrm{H}), 4.12-3.92(\mathrm{~m}, 2 \mathrm{H}), 3.85-3.65(\mathrm{~m}, 2 \mathrm{H}), 3.55-3.27(\mathrm{~m}, 2 \mathrm{H})$, 1.90-1.72 (m, 3H), 1.68-1.60 (m, 2H), 1.58-1.52 (m, 6H); IR (diamond) $\tilde{v}\left[\mathrm{~cm}^{-1}\right]: 3353\left(\mathrm{~m}, v_{\mathrm{N}-\mathrm{H}}\right), 2978\left(\mathrm{~m}, v_{\mathrm{C}-\mathrm{H}}\right), 2934(\mathrm{~m}$, $\left.v_{\mathrm{C}-\mathrm{H}}\right), 1715\left(w, v_{\mathrm{C}=\mathrm{O}}\right), 1521\left(s, v_{\mathrm{N}-\mathrm{H}}\right), 1455\left(s, v_{\mathrm{C}-\mathrm{H}(\mathrm{R}-\mathrm{CH} 3)}\right)$; DSC: $T_{\mathrm{g}}=125.9{ }^{\circ} \mathrm{C}$; GPC (THF): $\overline{M_{\mathrm{w}}}=8900 \mathrm{~g} / \mathrm{mol}, \overline{M_{\mathrm{n}}}=$ $6000 \mathrm{~g} / \mathrm{mol}, \mathrm{D}=1.48$.

\section{References}

1. Burkhart, A.; Fischer, J.; Mondrzyk, A.; Ritter, H. Macromol. Chem. Phys. 2014, 215, 421-425. doi:10.1002/macp.201300734

2. Mather, B. D.; Viswanathan, K.; Miller, K. M.; Long, T. E. Prog. Polym. Sci. 2006, 31, 487-531. doi:10.1016/j.progpolymsci.2006.03.001

3. Thöne, J.; Ritter, H. Macromol. Chem. Phys. 1987, 188, 2047-2059. doi:10.1002/macp.1987.021880904

4. Rehse, H.; Ritter, H. Makromol. Chem. 1989, 190, 697-706. doi:10.1002/macp.1989.021900403

5. Gormanns, M.; Rehse, H.; Ritter, H. Makromol. Chem. 1991, 192, 745-755. doi:10.1002/macp.1991.021920401

6. Gormanns, M.; Ritter, H. Tetrahedron 1993, 49, 6965-6974. doi:10.1016/S0040-4020(01)87972-2

7. Gormanns, M.; Ritter, H. Macromolecules 1994, 27, 5227-5228. doi:10.1021/ma00096a057

8. Carpino, L. A.; Parameswaran, K. N.; Kirkley, R. K.; Spiewak, J. W.; Schmitz, E. J. Org. Chem. 1970, 35, 3291-3295. doi:10.1021/jo00835a025

9. Carpino, L. A. Acc. Chem. Res. 1973, 6, 191-198. doi:10.1021/ar50066a003

10. Carpino, L. A.; Rice, N. W.; Mansour, E. M. E.; Triolo, S. A. J. Org. Chem. 1984, 49, 836-842. doi:10.1021/jo00179a017

11. Ohnishi, T.; Sugano, H.; Miyoshi, M. Bull. Chem. Soc. Jpn. 1972, 45, 2603-2607. doi:10.1246/bcsj.45.2603

\section{License and Terms}

This is an Open Access article under the terms of the Creative Commons Attribution License (http://creativecommons.org/licenses/by/2.0), which permits unrestricted use, distribution, and reproduction in any medium, provided the original work is properly cited.

The license is subject to the Beilstein Journal of Organic Chemistry terms and conditions:

(http://www.beilstein-journals.org/bjoc)

The definitive version of this article is the electronic one which can be found at: $\underline{\text { doi: } 10.3762 / \text { bjoc. } 12.26}$ 